\title{
Interstitielle Lungenerkrankungen
}

\section{Fotios Drakopanagiotakis}

Klinikum Fulda, Medizinische Klinik V, Fulda, Deutschland

\section{Epidemiologische Herausforderungen}

Die interstitiellen Lungenerkrankungen (interstitial lung diseases, ILDs) sind seltene Lungenerkrankungen, d.h. dass diese individuell weniger als 5 von 10000 Personen betreffen. Studien deuten auf eine Prävalenz der ILDs von 81/100 000 bei Männern gegenüber $67 / 100000$ bei Frauen hin. Ebenso ist die Gesamtinzidenz von ILD bei Männern (32/100 000/Jahr) etwas häufiger als bei Frauen (26/100 000/Jahr) und nimmt mit dem Alter zu. Eine sichere Unterscheidung der verschiedenen Formen der ILDs ist essenziell für robuste, qualitative epidemiologische Daten. Die genaue Zuordnung zu einer bestimmten Form einer ILD ist allerdings oft eine große Herausforderung, da ILDs nur eine begrenzte Anzahl radiologischer Muster aufweisen, die entsprechend unspezifisch sind.

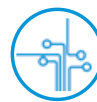

\section{Verbesserung des diagnostischen Verfahrens}

Fortschritte in der Bildgebung und in den bronchoskopischen Methoden können zu einer genauen Klassifikation mit relativ geringem Risiko für den Patienten mit einer ILD beitragen. Dank der hochauflösenden Computertomographie (high-resolution computed tomography, HRCT) des Thorax kann die Diagnose einer ILD oft nicht-invasiv gestellt werden. Die Standardisierung der transbronchialen Kryobiopsie und der Endobronchialen Ultraschall (EBUS)-Biopsie der mediastinalen/hilären Lymphknoten erlaubt die Diagnosesicherung ohne das Risiko einer chirurgischen Biopsie.

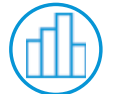

Register und Biobanken zu ILDs

Die Unterscheidung der verschiedenen Formen von ILDs bedarf ihrer genauen Phänotypisierung. In Register können Patienten mit ILDs eingeschlossen werden, die an randomisierten kontrollierten Studien aufgrund ihres Alters, Komorbiditäten oder atypischen radiologischen Charakteristika nicht teilnehmen dürfen. Register erweitern damit unsere Kenntnisse über die ILDs insbesondere über die sehr seltenen Formen der ILDs. Die Verknüpfung der Register mit Biobanken hat das Potenzial, durch das Verständnis von Krankheitsmechanismen individualisierte Therapien anbieten zu können.

\section{KARGER}

Fax +497614520714

information@karger.com

www.karger.com
(C) 2018 S. Karger GmbH, Freiburg

Accessible online at:

www.karger.com/kkp
Dr. Fotios Drakopanagiotakis

Klinikum Fulda

Medizinische Klinik V

Pacelliallee 4, 36043 Fulda, Deutschland

fotios.drakopanagiotakis@klinikum-fulda.de 